\title{
Deficiency of filaggrin regulates endogenous cysteine protease activity leading to impaired skin barrier function
}

\author{
X.W. Wang, ${ }^{1,2^{*}}$ J.J. Wang, ${ }^{1}$ D. Gutowska-Owsiak, ${ }^{2}$ M. Salimi, ${ }^{2}$ T.A. Selvakumar, ${ }^{2}$ A. Gwela, ${ }^{2}$ L.Y. \\ Chen, ${ }^{2}$ Y.J. Wang, ${ }^{2}$ E. Giannoulatou, ${ }^{3}$ G. Ogg $^{2 *}$ \\ ${ }^{1}$ State Key Laboratory of Military Stomatology, Department of oral medicine, School of Stomatology, The Fourth \\ Military Medical University, Xi'an Shaanxi Province, China \\ ${ }^{2}$ Medical Research Council Human Immunology Unit, Weatherall Insititute of Molecular Medicine, NIHR \\ Biomedical Research Centre, University of Oxford, Oxford, U.K. \\ ${ }^{3}$ Computational Biology Research Group, Weatherall Institute of Molecular Medicine, University of Oxford, Oxford, \\ U.K. \\ *Corresponding authors \\ Graham.ogg@ndm.ox.ac.uk and xinwen.wang@yahoo.com
}




\section{Summary}

Background. Atopic dermatitis (AD) is a common inflammatory skin disorder, characterized by skin barrier defects and enhanced allergen priming. Null mutations in the filaggrin gene are strongly associated with moderate-severe disease, but the pathways linking barrier dysfunction and cutaneous inflammation are still largely unknown.

Aim. To assess alteration of endogenous cysteine protease activity in filaggrin-deficient keratinocytes, and determine whether the alteration in cysteine protease activity affects epidermal barrier function and associated gene and protein expression.

Methods. We established a stable filaggrin knock down cell line and reconstructed epidermal equivalents in vitro. Barrier function of the reconstructed epidermis, barrier associated genes and proteins, and endogenous cysteine proteases activity were tested. Inhibitors of cysteine proteases were used to further evaluate the role of endogenous cysteine proteases in epidermal barrier function.

Results. Filaggrin knock down induced impaired epidermal barrier function. Microarray, Western blot and fluorescence staining showed reduced K10, ZO-1, E-cadherin, claudin-1 and occludin expression in filaggrin knock down keratinocytes. Compared with cysteine protease activity in control cells, protease activity was dramatically enhanced in filaggrin knock down keratinocytes. Furthermore, the administration of cysteine protease inhibitors significantly recovered K10 and tight junction protein expression, and the barrier defect induced by filaggrin deficiency.

Conclusions. This is the first observation of elevated endogenous cysteine protease activity in filaggrin-deficient keratinocytes, which may play an important role in impaired barrier function in $A D$ skin. Modulation of cysteine protease activity might be a novel therapeutic approach for $A D$ treatment.

Key words:

Atopic dermatitis; filaggrin; cysteine protease; barrier function 


\section{Introduction}

Atopic dermatitis (AD) is a common inflammatory skin disorder, which affects 15 to $30 \%$ of children and 2 to $10 \%$ of adults. ${ }^{1}$ It is characterized by skin barrier defect, enhanced allergen priming, susceptibility to cutaneous pathogen colonization, infection and inflammation driven by type 2 cells. $^{2}$ Although mutations in the filaggrin gene are considered as the most common causative genetic factor for the disease, the pathogenesis is still largely unknown.

Filaggrin is a keratinocyte protein contained within the upper epidermis. It is initially synthesized as a large, highly phosphorylated precursor, profilaggrin, which is stored in an inactive and insoluble form within keratohyalin granules. As keratinocytes undergo terminal differentiation, profilaggrin is dephosphorylated and cleaved into $37 \mathrm{kDa}$ filaggrin monomer molecules and a $32 \mathrm{kDa}$ profilaggrin $\mathrm{N}$-terminal domain (PND). ${ }^{3}$ Early research showed that filaggrin is degraded into amino acids and other components of the natural moisturizing factors, contributing to the retention of water within the stratum corneum, which is essential for homeostatic functions of the epidermis. ${ }^{4}$ Recently, it was also shown that profilaggrin degradation products are involved in many epidermal metabolic activities and may have more functions than previously anticipated..$^{5,6}$

The epidermis is a rich source of proteases that collectively display almost the full repertoire of catalytic chemistry. ${ }^{7}$ In recent years, defective regulation of proteases has emerged as a key contributor to diverse skin pathologies; $;^{7}$ abnormal protease activity ${ }^{8}$ or polymorphisms in genes encoding proteases and protease inhibitors have been identified as playing a role in atopic dermatitis pathogenesis. ${ }^{9}$ Of many proteases, cysteine proteases were shown have the closest link to atopic dermatitis. It has been confirmed that exogenous cysteine protease can directly disrupt epithelial tight junctions and activate keratinocytes, causing increased production of IL-6, IL-8, granulocyte-macrophage colony-stimulating factor (GM-CSF), and worsening AD severity. ${ }^{10}$

We hypothesized that filaggrin deficiency may influence endogenous cysteine protease activity of keratinocytes, disrupting the skin barrier and altering the susceptibility to transcutaneous allergen priming. In this study, we used immortalized keratinocytes (HaCaTs) as the targets for transduction and established a stable $F L G$ knock down cell line, assessed endogenous protease activity and effects on epidermis development and barrier function, in order to improve our understanding of the mechanisms underlying dysfunctional skin barrier in AD. 


\section{Methods}

\section{Generation of filaggrin knockdown HaCaT cells}

HaCaT cells (a kind gift from Prof Fusenig) were infected with lentiviral particles containing FLG shRNA (sc-43364-V) or empty vector (sc-108080, both Santa Cruz) using polybrene (Santa Cruz) according to the manufacturers' instructions. A stable clonal population was selected in puromycin-containing medium (at the concentration of $50 \mu \mathrm{g} / \mathrm{ml}$ ). Expression of filaggrin was examined by fluorescence staining, q-PCR and Western blot.

\section{Quantitative PCR analysis}

RNA was isolated using the RNEasy Mini kit (Qiagen) and qPCR was conducted using TaqMan RNA-to-CT 1 step kit (AB Applied Biosystems) according to manufacturers' protocols. TaqMan gene expression assays used were FLG (Hs00856927), and GAPDH (Hs0275899). Expression levels were measured in triplicate using ABI PRISM 7500 Fast sequence detection system (Applied Biosystems).

\section{Western blot}

Cell lysates were prepared in lysis buffer (Cell signaling). Equal amount of total proteins were loaded and run on SDS-PAGE. The gels were transferred to PVDF membranes using iBlot Dry Blotting System. After blocking, membranes were incubated overnight at $4^{\circ} \mathrm{C}$ with primary antibodies separately. Samples were used for immunoblotting with antibodies against filaggrin (Santa Cruz), keratin1 (Abcam), keratin5 (Lifespan biosciences), keratin10 (Epitomics), keratin14 (Epitomics), keratin17, E-cadherin (Epitomics), ZO-1 (Invitrogen), claudin-1 (Abcam), desmoplakin (Santa Cruz). Infrared dye-conjugated secondary antibodies (Li-Cor) were used for detection with Li-Cor scanner.

\section{Immunofluorescence staining}

Cells were cultured on slides, fixed with acetone for $5 \mathrm{~min}$, and stained as described previously ${ }^{11}$ using primary antibodies against filaggrin (Abcam), ZO-1 (Invitrogen), claudin-1 (Abcam), occludin (Invitrogen), catenin (Abcam). The PathScan Apoptosis and Proliferation Multiplex IF Kit (Cell Signaling) was used for proliferation and apoptosis assays. Confocal images were recorded on LSM710 microscope equipped with a Zeiss63 objective. The mean fluorescence intensity was measured by Image J software.

\section{Epidermis equivalents and thickness measurement}

$2 \times 10^{5}$ knockdown HaCaTs and vector control cells were seeded into culture inserts (Millipore), cultured in DMEM supplemented with $10 \%$ FCS. After 3 days of submerged culture, cells were raised to the air-liquid interface to produce multilayer structure and fed as required. Samples were collected after ten days of culture. Four independent experiments were undertaken. Sections $5 \mu \mathrm{m}$ thick were stained with hematoxylin and eosin (H\&E) for histological observation. The thickness of the epidermis was measured at three different sites using Image J.

\section{Electrical resistance}

Measurements of transepithelial electrical resistance (TER) were conducted using EVOM instrument. All measurements of TER are reported as background-corrected values taking into account the resistance measured across an empty insert containing appropriate media.

\section{Microarray assay}

RNA was isolated as described above. Microarray acquisition was performed by Service XS (Holland, Leiden) on Illumina HT12 BeadArray platform; the experiment was set up in biological triplicates. Expression of filaggrin transcript was assessed based on the hybridization to the Illumina probe ILMN_2134130.

\section{Protease activity assay}

General endogenous cysteine proteases and cathepsin B activity were measured respectively using the Protease Assay Kit (Calbiochem) and Cathepsin B activity assay kit (Calbiochem) according to manufacturer's protocol. 
Briefly, the cell pellet was washed with ice-cold PBS, cell lysis buffer was added, and then incubated on ice for 30 min. Supernatant was collected. After protein concentration adjustment, fluorescence was measured.

\section{Application of inhibitor of cysteine proteases}

General cysteine protease inhibitor E64 and cathepsin B inhibitor CA074 were used in the study. FLG knock down

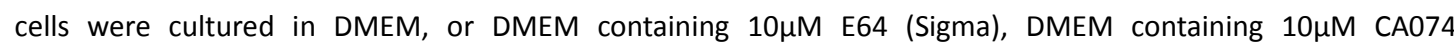
(Calbiochem) respectively. Medium changes were performed every two days. After one week, cells were collected for Western blot analysis.

Organotypic epidermal models were prepared according to the above protocols. The medium used for 3D

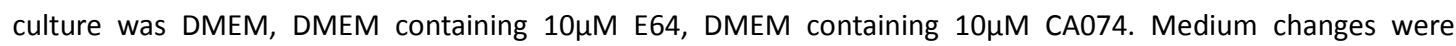
performed every day. The measurements of transepidermal electrical resistance (TER) were performed when models were raising to the air-liquid interface, and the third, sixth day after cells were raised to the air-liquid interface. After ten days of culture, the samples were harvested for staining and measurements.

\section{Statistical analysis}

The student's t-test was used for comparisons of differences between groups. ${ }^{*} p<0.05$ was considered statistically significant, ${ }^{* *} p<0.01$ was considered highly significant. Microarray data were normalized using lumi ${ }^{12}$ analysed with LIMMA. ${ }^{13}$ After multi comparison correction genes were considered differentially expressed when adjusted $p<0.05$, and $\log _{2} F C<-0.5$ or $>0.5$. 


\section{Results}

\section{FLG knockdown promotes cell proliferation}

Filaggrin knock down in HaCaTs was confirmed by qPCR, fluorescent staining and Western blot analysis (Fig. 1a-c). Proliferation and apoptosis assays showed that the percentage of proliferating cells in the filaggrin-knockdown group (8.90\%) is significantly higher than that of the control group (3.01\%). The percentage of apoptotic cells appeared to be reduced in knockdown cells, but this did not reach significance (Fig. 1d).

\section{FLG knock down results in generation of thicker epidermis but impairs barrier function}

FLG knock down HaCaTs (KD) became seven to eight cells deep and made a stratified layer with morphological differentiation. The average epidermis thickness was $57.1 \mu \mathrm{m}$, and was significantly increased compared to vector control transfected HaCats (43.3um, $P<0.05$; Fig. 2a, b). However, a sparse matrix could be more easily observed in filaggrin knock down cells.

Transepithelial electrical resistance (TER) was used to determine the barrier function of the reconstructed epidermis. Under symmetrical culture condition, the epidermis reconstructed with filaggrin knock down cells exhibited significantly lower TER (a mean TER of $26.3 \Omega / \mathrm{cm}^{2}$ ) compared with the control group (the mean TER of control group was $44.3 \Omega / \mathrm{cm}^{2}$ ) (Fig. 2c).

\section{Most tight junction and keratin genes were not disturbed in FLG knock down keratinocytes}

Microarray analysis was carried out to examine the effect of $F L G$ knockdown on tight junction and keratin gene expression profile. 26 tight junction genes and 26 keratin genes were detected and compared between filaggrin knock down cells and control cells. The results showed that K1 and K17 were downregulated while the expression of other keratin and tight junction genes were preserved in filaggrin knock down cells (Supplementary Table 1,2).

Western blot and fluorescence staining was performed to further verify the above result; ZO-1, E-cadherin, claudin-1, desmoplakin, KRT1/KRT10, KRT5/KRT14 and KRT17 protein expression were tested. Consistent with the microarray data, KRT1 and KRT17 expression were down-regulated. However, it was notable that KRT10, ZO-1, E-cadherin, claudin-1 and occludin protein levels in filaggrin knock down cells were significantly down regulated at the protein level (Fig. 3).

\section{Cysteine protease activity is enhanced in FLG knock down keratinocytes}

General endogenous cysteine protease and cathepsin B activity were measured. The results showed that endogenous cysteine protease activity in filaggrin knock down cells was 1982.15 IFU, and cathepsin B activity was $12158.6 \mathrm{IFU}$, in contrast with that in control cells (endogenous cysteine protease activity: 1729.9 IFU, cathepsin B activity: 10537.1 IFU) (Fig. 4). The filaggrin knock down cells had a $14.6 \%$ increase in endogenous cysteine proteases activity and $15.3 \%$ increase in cathepsin B activity.

Cysteine protease inhibitors recover epidermal barrier function defect induced by FLG deficiency

To determine the effect of elevated cysteine protease activity on barrier function, cysteine protease inhibitors were used in cultured epidermal equivalents. The results showed that the epidermal matrix was more condensed, and the TER was elevated after cysteine protease 
inhibitor administration. Moreover, we noted the difference between the inhibitors used, namely E64 provided better restoration of the barrier function compared to CA074, consistent with the mode of action(Fig. 5).

Cysteine protease inhibitors up regulated some keratin and tight junction protein expression In order to test whether the decreased keratin and tight junction protein expression in filaggrin knock down HaCaTs was a result of cysteine protease activity alteration, KRT1, KRT10, KRT17, E-cadherin, ZO-1, claudin-1, occludin and catenin protein level were tested again after cysteine protease inhibitor administration. The results showed that cysteine protease inhibitor E64 significantly recovered ZO-1, claudin-1, E-cadherin and occludin protein expression in knock down cells (Fig. 6). 


\section{Discussion}

In this study, we established a stable filaggrin (FLG) knock down cell line, which made it possible to analyze pathomechanisms linked to filaggrin deficiency. The FLG knock-down cells showed increased proliferation and less apoptosis, which was consistent with previous studies. In 2001, Presland et al generated a keratinocyte cell line that expressed human filaggrin, and showed that filaggrin expression lead to reduced keratinocyte proliferation ${ }^{14}$. Recently, a study on the $\mathrm{N}$-terminal fragment of profilaggrin (PF-N) released during profilaggrin degradation showed that PF-N possessed the ability to regulate keratinocyte proliferation and differentiation ${ }^{15}$, which supports the findings presented herein. We used the FLG knock-down cell line to generate reconstructed epidermis equivalents, which showed that although thicker epithelium developed in the knock-down cells compared to the control cells, transepithelial resistance assay showed that the barrier function of three-dimensional epidermis reconstructed with filaggrin deficient keratinocytes was impaired. The results correspond well to previously described findings in the skin of AD patients. ${ }^{16}$

Tight junctions and keratins are considered to be important for barrier function of the skin. We detected altered profile of keratin expression, with K1 and K17 mRNA significantly altered in filaggrin knock down cells. Interestingly, although no obvious change was observed in K10 mRNA expression and tight junctions mRNA levels, Western blot and Immunofluorescence staining showed that keratin 10 (K10) and expression of some tight junction proteins including ZO-1, E-cadherin, claudin-1 and occludin were reduced, suggesting a post-translational effect, and these proteins may be degraded in special circumstances.

Epidermal homeostasis is highly dynamic with proteolytic activities being essential. An increasing body of evidence showed that these proteases are more than scavengers, with changes in the proteolytic balance of the skin resulting in different pathological conditions, such as epidermal hyperplasia, ${ }^{17}$ skin inflammation ${ }^{18}$ and cancer metastasis. ${ }^{19}$ Cysteine proteases are papain-like peptidases and mainly localize in the acidic cellular compartments, participating in degradation of intracellular or endocytosed proteins. ${ }^{20}$ They therefore take part in multiple biological functions, including being implicated in the antigen processing and presentation pathways. ${ }^{21}$ For example, cathepsin B, C, F, H, L, K O, S, V have been identified in humans..$^{22}$ It was shown that cysteine proteases have direct proteolytic activity on skin extracellular matrix and degrade tight junction proteins, ${ }^{7}$ which contributes to barrier impairment and delayed barrier recovery. They can also affect other protease activities and, therefore, influence skin barrier function indirectly. ${ }^{23}$

In this study, we first assessed the endogenous cysteine protease activity of filaggrin deficient keratinocytes and documented that general cysteine protease and cathepsin B activity in filaggrin knock down keratinocytes is dramatically higher than that in control cells (Fig. 4), although no significant change was observed on the mRNA level. Highly specific inhibitors designed based on the tertiary structures of cysteine proteases have been widely used. E64 is an epoxysuccinate peptide derivative which is a general cysteine protease inhibitors, ${ }^{24}$ whereas CA074 is a specific inhibitor that selectively inhibits the activity of cathepsin B in vivo as well as in vitro. ${ }^{24}$ In order to further evaluate the effect of cysteine activity on barrier function, E64 and CA074 were used in our study. The results indicated that the administration of cysteine protease inhibitors restored barrier function to different degrees (Fig. 5), confirming the role of cysteine proteases activity in 
barrier function. Western blot and fluorescent analysis showed that cysteine protease inhibitors partly up-regulated keratin expression, and significantly promoted E-cadherin, ZO-1, claudin and occludin protein expression (Fig. 6), which may contribute to barrier function restoration. There was no statistical significant difference between E64 and CA074, which might attribute to overlapping roles of E64 and CA074.

Cystatins are the predominant inhibitors of cysteine proteases in the epidermis. ${ }^{25}$ They demonstrated strong inhibition not only of endogenous cysteine protease, but also bacterial cysteine protease and virus cathepsins, therefore showing strong protection from bacterial and viral infections of the skin. ${ }^{26}$ Previous studies showed that filaggrin, expressed as a late keratinocyte protein contained within the granular layer of the upper epidermis can be conjugated with cystatins. Whether the filaggrin conjugation influences the function of cystatins, further affecting endogenous cysteine protease activity needs to be further investigated. Our previous study showed filaggrin can inhibit house dust mite-derived phospholipases and reduce $T$ cell responses specific to endogenous lipids presented by CD1a, ${ }^{5}$ so we also cannot exclude the possibility that filaggrin directly or indirectly inhibits endogenous cysteine protease directly.

\section{Conclusion}

In summary, the current report is the first description of elevated endogenous cysteine protease activity in filaggrin deficient keratinocytes. Our study showed that the raised endogenous cysteine protease activity may play an important role in barrier dysfunction. Future therapeutic interventions may target this pathway. 


\section{Acknowledgements}

The study was supported by National Natural Science Foundation of China (grant number: 81371154), Key Science Technology Program Shaanxi Province, China (grant number: 2015SF150), UK Medical Research Council and NIHR Biomedical Research Centre Programme. 


\section{What's already known about this topic?}

- Atopic dermatitis (AD) is a common inflammatory skin disorder characterized by skin barrier defects and susceptibility to cutaneous pathogen colonization.

- Mutations in the filaggrin gene are a causative genetic factor for AD.

What does this study add?

- Keratinocyte endogenous cysteine protease activity is elevated in the setting of filaggrin deficiency, and influences epidermal barrier function.

- Modulation of cysteine protease activity might be a novel therapeutic approach for $A D$ treatment. 


\section{References}

1. Asher MI, Montefort S, Bjorksten B, Lai CK, Strachan DP, Weiland SK, Williams H: Worldwide time trends in the prevalence of symptoms of asthma, allergic rhinoconjunctivitis, and eczema in childhood: ISAAC Phases One and Three repeat multicountry cross-sectional surveys, Lancet 2006, 368:733-743

2. Boguniewicz M, Leung DY: Recent insights into atopic dermatitis and implications for management of infectious complications, J Allergy Clin Immunol 2010, 125:4-13; quiz 14-15

3. Resing KA, Walsh KA, Dale BA: Identification of two intermediates during processing of profilaggrin to filaggrin in neonatal mouse epidermis, J Cell Biol 1984, 99:1372-1378

4. Scott IR, Harding CR: Filaggrin breakdown to water binding compounds during development of the rat stratum corneum is controlled by the water activity of the environment, Dev Biol 1986, 115:84-92

5. Jarrett R, Salio M, Lloyd-Lavery A, Subramaniam S, Bourgeois E, Archer C, Cheung KL, Hardman C, Chandler D, Salimi M, Gutowska-Owsiak D, Bernardino de la Serna J, Fallon PG, Jolin H, McKenzie A, Dziembowski A, Podobas EI, Bal W, Johnson D, Moody DB, Cerundolo V, Ogg G: Filaggrin inhibits generation of CD1a neolipid antigens by house dust mite-derived phospholipase, Sci Transl Med 2016, 8:325ra318

6. Yoneda K, Nakagawa T, Lawrence OT, Huard J, Demitsu T, Kubota Y, Presland RB: Interaction of the profilaggrin N-terminal domain with loricrin in human cultured keratinocytes and epidermis, J Invest Dermatol 2012, 132:1206-1214

7. de Veer SJ, Furio L, Harris JM, Hovnanian A: Proteases: common culprits in human skin disorders, Trends Mol Med 2014, 20:166-178

8. Albanesi C: Keratinocytes in allergic skin diseases, Curr Opin Allergy Clin Immunol 2010, 10:452-456

9. Liang Y, Chang C, Lu Q: The Genetics and Epigenetics of Atopic Dermatitis-Filaggrin and Other Polymorphisms, Clin Rev Allergy Immunol 2015,

10. Meyer-Hoffert U: Reddish, scaly, and itchy: how proteases and their inhibitors contribute to inflammatory skin diseases, Arch Immunol Ther Exp (Warsz) 2009, 57:345-354

11. Wang X, Liu Y, Deng Z, Dong R, Hu S, Li Y, Jin Y: Inhibition of dermal fibrosis in self-assembled skin equivalents by undifferentiated keratinocytes, J Dermatol Sci 2009, 53:103-111

12. Du P, Kibbe WA, Lin SM: lumi: a pipeline for processing Illumina microarray, Bioinformatics 2008, 24:1547-1548

13. Smyth GK: Linear models and empirical bayes methods for assessing differential expression in microarray experiments, Stat Appl Genet Mol Biol 2004, 3:Article3

14. Presland RB, Kuechle MK, Lewis SP, Fleckman P, Dale BA: Regulated expression of human filaggrin in keratinocytes results in cytoskeletal disruption, loss of cell-cell adhesion, and cell cycle arrest, Exp Cell Res 2001, 270:199-213

15. Aho S, Harding CR, Lee JM, Meldrum H, Bosko CA: Regulatory role for the profilaggrin N-terminal domain in epidermal homeostasis, J Invest Dermatol 2012, 132:2376-2385

16. Schmuth M, Blunder S, Dubrac S, Gruber R, Moosbrugger-Martinz V: Epidermal barrier in hereditary ichthyoses, atopic dermatitis, and psoriasis, J Dtsch Dermatol Ges 2015, 13:1119-1123

17. Nishimura F, Naruishi H, Naruishi K, Yamada T, Sasaki J, Peters C, Uchiyama Y, Murayama Y: Cathepsin-L, a key molecule in the pathogenesis of drug-induced and I-cell disease-mediated gingival 
overgrowth: a study with cathepsin-L-deficient mice, Am J Pathol 2002, 161:2047-2052

18. Steinhoff M, Buddenkotte J, Shpacovitch V, Rattenholl A, Moormann C, Vergnolle N, Luger TA, Hollenberg MD: Proteinase-activated receptors: transducers of proteinase-mediated signaling in inflammation and immune response, Endocr Rev 2005, 26:1-43

19. Szpaderska AM, Frankfater A: An intracellular form of cathepsin B contributes to invasiveness in cancer, Cancer Res 2001, 61:3493-3500

20. Reinheckel T, Peters C, Kruger A, Turk B, Vasiljeva O: Differential Impact of Cysteine Cathepsins on Genetic Mouse Models of De novo Carcinogenesis: Cathepsin B as Emerging Therapeutic Target, Front Pharmacol 2012, 3:133

21. Chapman HA, Riese RJ, Shi GP: Emerging roles for cysteine proteases in human biology, Annu Rev Physiol 1997, 59:63-88

22. Liu J, Sukhova GK, Sun JS, Xu WH, Libby P, Shi GP: Lysosomal cysteine proteases in atherosclerosis, Arterioscler Thromb Vasc Biol 2004, 24:1359-1366

23. Halangk W, Lerch MM, Brandt-Nedelev B, Roth W, Ruthenbuerger M, Reinheckel T, Domschke W, Lippert H, Peters C, Deussing J: Role of cathepsin B in intracellular trypsinogen activation and the onset of acute pancreatitis, J Clin Invest 2000, 106:773-781

24. Katunuma N, Matsunaga Y, Himeno K, Hayashi Y: Insights into the roles of cathepsins in antigen processing and presentation revealed by specific inhibitors, Biol Chem 2003, 384:883-890

25. Rawlings ND, Morton FR, Barrett AJ: MEROPS: the peptidase database, Nucleic Acids Res 2006, 34:D270-272

26. Katunuma N: Posttranslational processing and modification of cathepsins and cystatins, J Signal Transduct 2010, 2010:375345 


\section{Figure legends}

Figure 1 Generation of filaggrin knockdown clones of HaCaT keratinocytes. (a) Fluorescence staining of filaggrin stable knockdown cells and vector control cells with antibody to filaggrin (green) and DAPI nuclear stain (blue). (b) q-PCR analysis of filaggrin in knockdown and control cells. (c) Western blot analysis of knockdown and the control cells. The mean intensity is shown in the graph on the right. (d) FLG knockdown cells and control cells were stained with the PathScan Apoptosis and Proliferation Multiplex IF Kit for tubulin (red), phospho-Histone H3 (green) and cleaved PARP (blue) to show proliferating ability and cell apoptosis. Graphical representation of the results show the percentage of proliferating cells and the percentage of apoptotic cells respectively ( 3000 cells assessed). ${ }^{* *} p<0.01$, Scale bars: $100 \mu \mathrm{m}, \mathrm{n}=3$. (e) Hematoxylin and eosin (HE) staining of organotypic epidermis constructed with knockdown keratinocytes and control cells, with the darker zone at the base representing the scaffold. Scale bars $100 \mu \mathrm{m}$. (f) Epithelial thickness was measured in the KD group compared to that in control group, $n=5$. (g) Transepithelial resistance (TER) of organotypic epidermis constructed with KD cells and control cells was measured, $\mathrm{n}=5 .{ }^{*} p<0.05,{ }^{* *} p<0.01$.

Figure 2 Keratin and tight junction proteins expression in filaggrin knock down HaCaTs. (a) Western blot analysis of K1, K5, K10, K14 and K17 in knock down cells and the control cells. The mean intensity was calculated and shown in the graph on the right-hand side, $n=5$. (b) Western blot analysis of ZO-1, E-cadherin, claudin-1 and desmoplakin in filaggrin knock down cells and control cells. The mean intensity was calculated and shown in the graph on the right-hand side. Scale bars: $50 \mu \mathrm{m}, \mathrm{n}=5$. (c) Fluorescence staining analysis of keratins and tight junction proteins. The mean fluorescent intensity was measured and shown in the graph on the right. ${ }^{*} p<0.05$, $* * p<0.01$.

Figure 3 Endogenous cysteine protease activity of filaggrin knock down HaCaTs and the effect on skin barrier function. (a) Overall endogenous cysteine protease activity of KD cells and control cells. (b) Endogenous cathepsin B activity of KD cells and control cells. All assays were performed 5 times independently, and the values were showed as mean fluorescence unit $\pm S$.D. (c) Morphological change of organotypic epidermis after

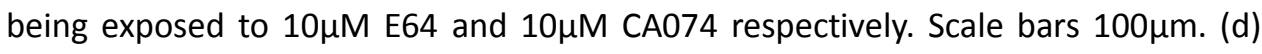
Transepithelial electrical resistance of organotypic epidermis after being exposed to

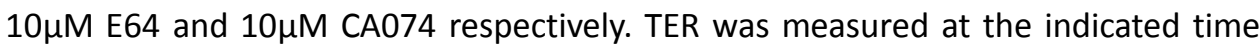
points, $\mathrm{n}=5$. ${ }^{*} p<0.05, * * * p<0.001$.

Figure 4 Western blot and fluorescence staining analysis of keratins and tight junction proteins expression after exposure to protease inhibitors. (a) Western blot analysis of K1, K10, K17, E-cadherin, ZO-1 and claudin-1 expression. The mean intensity was calculated and shown in the graph on the right-hand side, $n=5$. (b) Fluorescence staining analysis of ZO-1, claudin-1, occludin and catenin expression. The mean fluorescent intensity was calculated and shown in the graph on the right, $\mathrm{n}=5, * p<0.05$, 
$* * \mathrm{P}<0.01$

Supplementary table 1

Differences in tight junction gene expression of filaggrin knockdown $\mathrm{HaCaT}$ keratinocytes versus control cells as reported by mRNA microarray profiling. All experiments were performed in triplicates. The genes with a minimum log-fold change $(\log 2 \mathrm{FC})$ of \pm 0.5 and $p$-value lower than 0.05 were classified as differentially expressed and marked with *.

Supplementary table 2

Difference in keratin genes expression of FLG knock down HaCaT keratinocytes versus control cells. All experiments were performed with triplicates. The genes with a minimum log-fold change $(\log 2 \mathrm{FC})$ of \pm 0.5 and $p$-value lower than 0.05 were classified as differentially expressed and marked with *. 